\title{
Coupled Plasma Filtration Adsorption: A Multipurpose Extracorporeal Detoxification Therapy
}

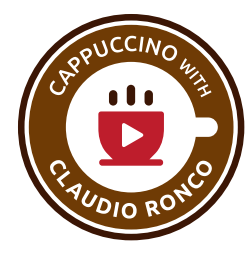

\author{
Gaetano La Manna Gabriele Donati \\ Nephrology Dialysis and Renal Transplantation Unit, S. Orsola University Hospital, Bologna, Italy
}

\section{Keywords}

Coupled plasma filtration adsorption - Detoxification .

Sepsis · Liver failure · Rhabdomyolysis · Poisoning

\begin{abstract}
Coupled plasma filtration adsorption (CPFA) is a detoxification system that combines a plasma adsorption circuit with a continuous renal replacement therapy. The circuit consists of a plasma filter, a resin/adsorbent cartridge and a haemofilter. It differs from many other types of extracorporeal therapies in that the upper part of the circuit can be considered a "closed loop". In this manner, the plasma separated by the plasma filter passes through an adsorbent cartridge containing a resin with high affinity to many cytokines, mediators and toxins/poisons. After passing through the cartridge, the purified plasma is returned to the patient. The second part of the circuit, the haemofilter, can then be used to remove small toxins that are not adsorbed by the resin or to modulate the patient hematic volume. Although more complex, the use of a plasma-separation step prior to the passage through the resin cartridge offers advantages over adsorption by haemoperfusion. The plasma passes through the resin cartridge with a lower velocity than the blood flow, and this allows better contact time for the toxins with the resin, and more thorough penetration into the resin pores. The adsorption of many toxins is highly dependent on a factor expressed as linear velocity.
\end{abstract}

In addition, the plasma does not contain cells or a very limited number of platelets, allowing less potential activation upon contact with the resin. Although the technique was originally developed for the treatment of sepsis and septic shock, there are many additional applications where there can be an advantage of having access to larger molecular weight toxins (compared to haemofiltration) and avoiding the loss of important physiologic substances such as albumin. Some of these new applications, in patients with or without acute kidney injury, include liver failure, rhabdomyolysis, severe autoimmune exacerbations and poisonings. In this article, we discuss some of the basic principles involved in sorbent technology, and how these may contribute to treatment efficacy, review the actual experiences with CPFA and finally discuss the results of recent human studies and their implications. Video Journal Club "Cappuccino with Claudio Ronco" at http://www.karger.com/?doi=490234.

(c) 2018 S. Karger AG, Basel

\section{Introduction}

Coupled plasma filtration adsorption (CPFA) is an extracorporeal blood purification treatment, which combines a first stage of plasma separation and adsorption of cytokines, inflammatory mediators and/or toxins, followed by a second stage of haemofiltration for

\section{KARGER}

(c) 2018 S. Karger AG, Basel

E-Mail karger@karger.com

www.karger.com/bpu
Gaetano La Manna, MD, PhD

Nephrology Dialysis and Renal Transplantation Unit

S.Orsola University Hospital

Via Massarenti 9, IT-40138 Bologna (Italy)

E-Mail gaetano.lamanna@unibo.it 
Fig. 1. The CPFA circuit consists of a Micropes $^{\mathrm{TM}}$ plasmafilter $\left(0.45 \mathrm{~m}^{2}\right)$ in series with a high permeability polyphenylene haemofilter (Kuf $41 \mathrm{~mL} / \mathrm{h} / \mathrm{mm} \mathrm{Hg}$, surface area $1.4 \mathrm{~m}^{2}$ ). The plasma flow rate is 30 $40 \mathrm{~mL} / \mathrm{min}$ and the plasma passes into the sorbent adsorption cartridge. The cartridge contains a 70-gram styrenic polymer resin. The resin is composed of mesoporous beads; the bead size is $50-100 \mu \mathrm{m}$; the average pore diameter is $30 \mathrm{~nm}$ and the surface area is $700 \mathrm{~m}^{2} / \mathrm{g}=50,000 \mathrm{~m}^{2}$.

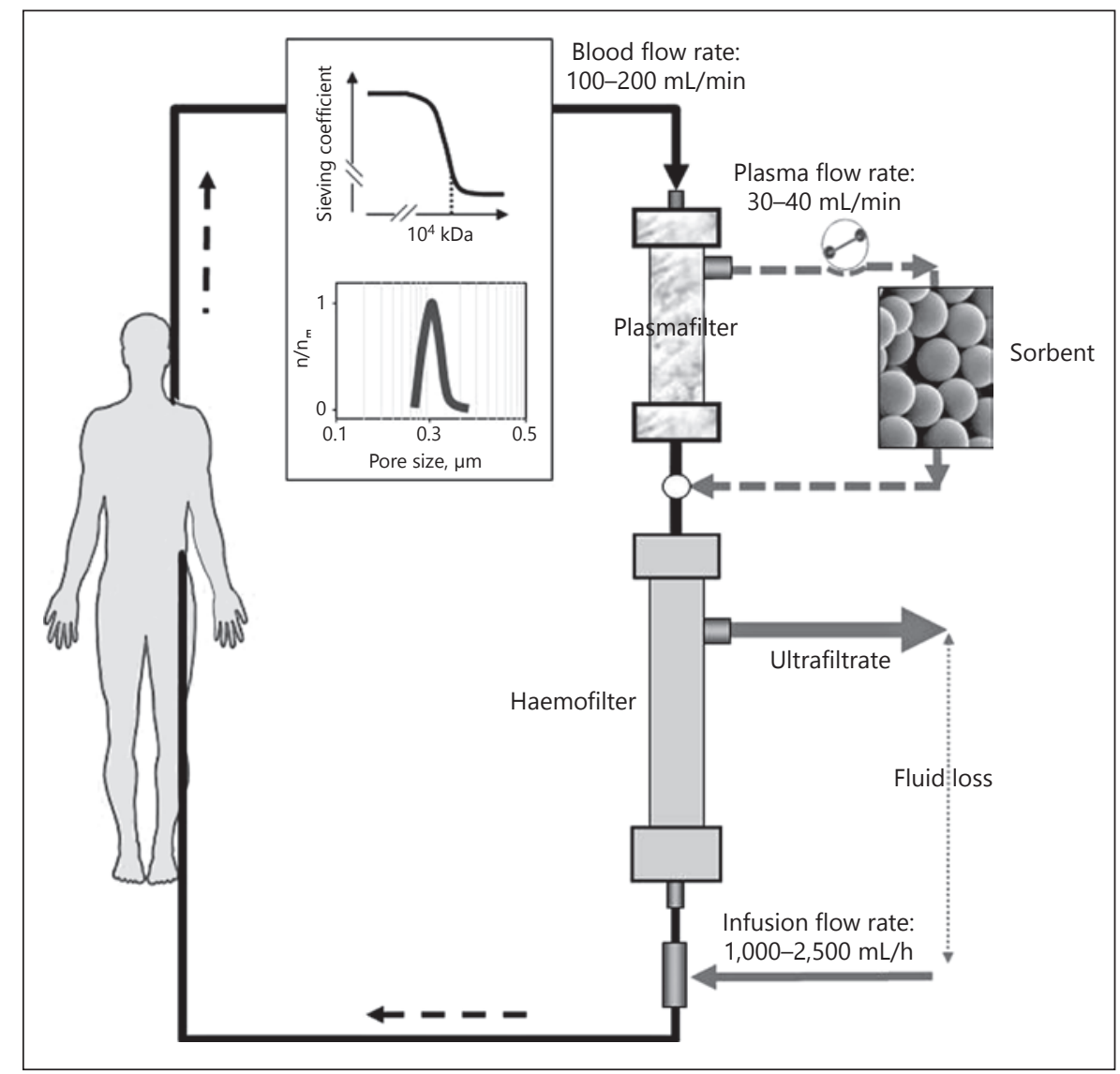

volume control and removal of small water-soluble mediators (Fig. 1).

CPFA was originally developed as a treatment for sepsis in the mid-1990s to address the need to remove inflammatory mediators and cytokines that are not easily or effectively removed by conventional extracorporeal methods (haemodialysis, haemodiafiltration, plasma exchange) [1].

The technique later evolved to include more generalized applications to remove other inflammatory mediators and toxins in clinical situations such as liver failure, rhabdomyolysis and cytokine response during severe non-infectious systemic anti-inflammatory response syndrome (SIRS) and poisoning. It offers the advantage of better extracorporeal depurations compared to traditional renal replacement therapies, since the increased pore size of the plasma membrane permits passage of large cytokines and mediators, which normally would not be possible with traditional haemodialysis membranes, with subsequent adsorption to the hydrophobic resin. It also offers an advantage over high permeability, high volume or plasma exchange in that there is a minor loss of albumin, protein or many important physiologic components, since many of these molecules are not adsorbed by the resin and can be reinfused back to the patient.

In 2003, Ronco et al. [2] proposed to use CPFA to remove pro- and anti-inflammatory mediators in a nonselective manner using the concept of the "peak concentration hypothesis" to remove excess peaks of soluble mediators found in the blood. He also suggested that in addition to conventional continous veno venous hemofiltration $(\mathrm{CVVH})$, that high volume or high permeability or adsorption may be alternative techniques to augment the removal of these excess cytokine peaks [2]. Although blood purification was used in animal experiments as early as 1948, growth was limited by technology. The concept of using extracorporeal techniques in critically ill patients for simultaneous and non-selective removal of many cytokines involved in the septic process gave rise to a new era in blood purification. This generated many new technologies and devices with a renewed interest to use extracorporeal therapy as a tool for immunomodulation and went far beyond the traditional use of only providing renal support for small toxin removal or volume control. 
At that time, cytokines were often considered pro-inflammatory or anti-inflammatory and a hypothesis emerged that there were 2 main processes that were driving the inappropriate immune/inflammatory response: a (SIRS and a compensatory anti-inflammatory response syndrome [CARS]) [3]. Both were considered detrimental to the patient response. SIRS was thought to play an important role in generating a strong inflammatory response that further augmented coagulation disorders, oxygen radical production all leading to organ dysfunction, while CARS was thought to be important in suppressing the appropriate immune response to fight infection.

As the understanding of the pathophysiology of sepsis continued to grow, the complexity of sepsis became much clearer [4]. SIRS and CARS probably do play a role but in a much more complex intertwined manner. Cytokines and inflammatory mediators need to be considered in the context of more than "pro" or "anti" inflammatory, but rather are they produced appropriately at the right concentration, right location and at the right time. Despite this more complex role, excess cytokines do seem to play a role in the clinical consequences of sepsis. Several studies have shown that a sustained excess production of both types of cytokines that were typically classified as either pro- or anti-inflammatory are associated with increased morbidity and mortality [5].

Recently, there has been a renewed interest to evaluate whether the use of adsorption may offer depurative benefits by the possibility to adsorb larger cytokines/mediators associated with certain non-septic, non-acute kidney injury (AKI) indications such as myoglobin for patients with severe rhabdomyolysis, removal of toxins associated with liver dysfunction/failure or elimination of poisons or drug overdoses when these have limited removal by haemodialysis or haemodiafiltration.

There are many triggers for inflammation in critically ill patients and both sepsis and sterile systemic inflammation share many inflammatory pathways. In bacterial sepsis, pathogen-associated molecular patterns interact with pattern recognition receptors, including toll-like receptors.

Sterile inflammation includes conditions such as severe non-penetrating poly-trauma, ischaemia-reperfusion injury and haemorrhagic shock. In these circumstances, endogenous molecules, such as DNA, histones and heat shock proteins are released upon cell damage or necrosis. These are called danger-associated molecular patterns and trigger the inflammatory response by many of the same pattern recognition receptors as in sepsis [5].
In both sepsis and sterile inflammation, there is a balance between an appropriate and dysregulated inflammatory response. Cytokines are important in the host response to neutralize bacteria and are essential for the repair process of damaged tissue. An excess hyper-productive inflammatory response, as well as the simultaneous production of cytokines that attenuate the response can lead to an excess of cell activation, inappropriate microbial inactivation, further activation of the inflammatory cascade, oxygen radical mediated damage and organ dysfunction [6].

Dysfunction of a single organ system and subsequent productions of toxins or mediators can also lead to a dysregulation or dysfunction of other organ systems. An example of this is the production of high levels of bilirubin and toxic fatty acids during acute hepatic failure. These toxic hydrophobic molecules can bind to liver-associated fatty acid binding protein and subsequently lead to cast nephropathy or nephrotoxicity, since they are filtered by glomeruli and internalized by proximal tubule cells probably by megalin-mediated endocytosis [7]. This highlights the importance of a better understanding of pathophysiologic mechanisms of organ damage, and what molecules may be useful to target with adsorptive therapies.

\section{CPFA as a Therapeutic Tool}

Extracorporeal therapies use different approaches for depuration including diffusion, convection, adsorption or plasma exchange. The open questions however are as follows: Which therapy should be used? When should the therapy be started? Which patients will benefit? What cytokines are beneficial to remove? How can you avoid the loss of beneficial substances? How can you evaluate whether the therapy is effective?

Most cytokines and mediators implicated in the pathophysiology of sepsis and organ dysfunction during critical illness are too large to be removed efficiently with conventional haemodialysis or haemofiltration. It is important to consider not only the cytokine molecular weight but also the molecular dimension (or essentially the shape of the molecule as it rotates in solution), commonly expressed as the Stokes-Einstein radius. The molecular dimensions of these cytokines do not pass easily through the haemodialyzer pores. This limits removal by the barrier created between the cytokine in the blood compartment and the haemodialyzer membrane, even in the presence of high convective volumes. If the cytokine does not pass through the membrane pores, it will remain on the 
Fig. 2. Molecular weight of cytokines and chemochines during septic conditions, modified from Bello et al. [35]. IL, Interleukin.

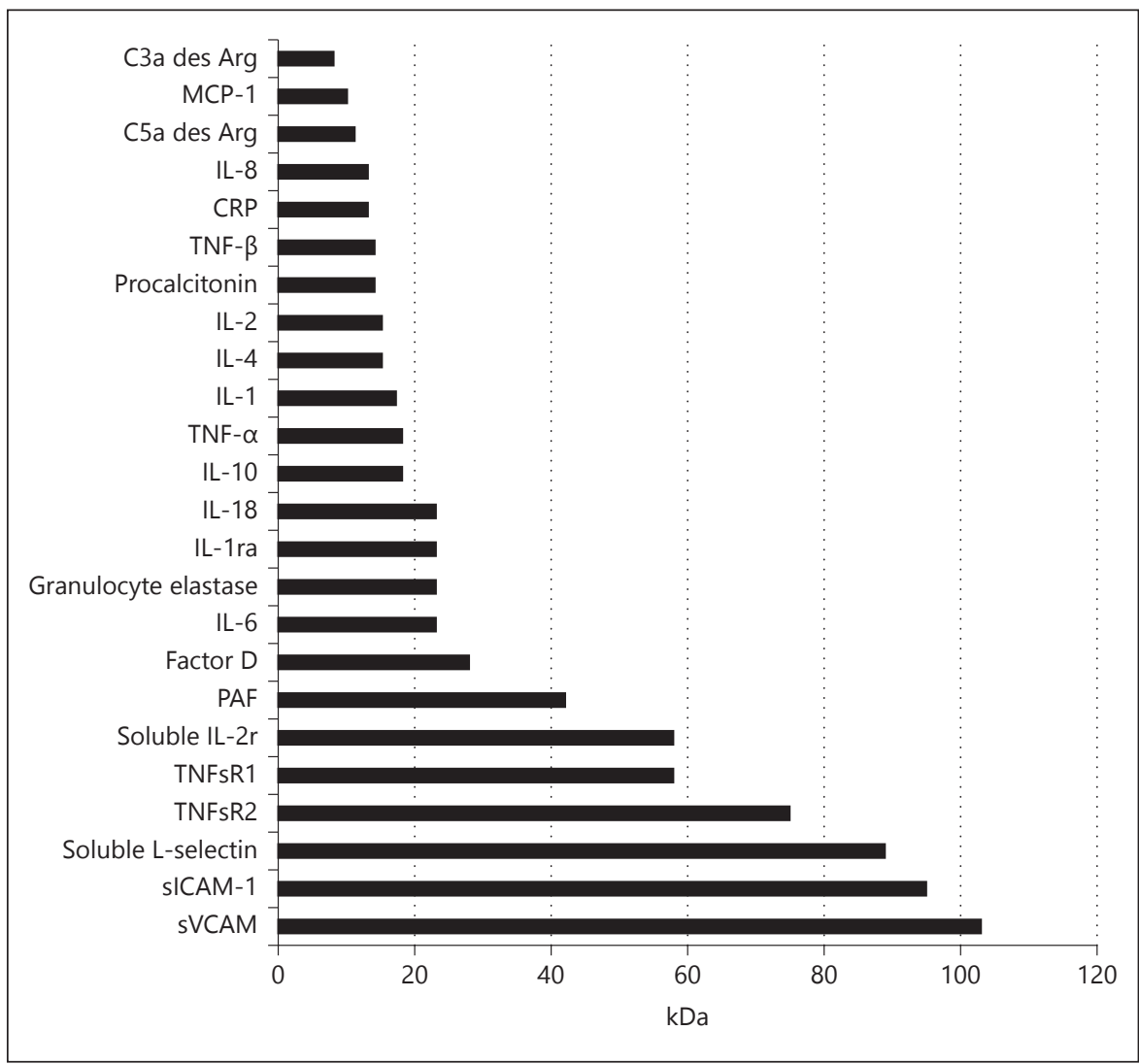

blood side even if great quantities of plasma water are removed by convection.

High permeability dialyzers were introduced to increase the removal of larger cytokines, but have the disadvantage of being able to be used for only short periods of time due to the risk of losing large quantities of albumin and other important physiologic substances.

Haemo- and plasma perfusion, where either blood or plasma pass through an adsorptive cartridge, offer the possibility to bind to an adsorptive matrix and selectively remove either specific mediators or toxins, or classes of mediators based on particular properties, such as hydrophobicity. These also offer distinct advantages in that the surface area of the adsorbent matrix can be quite large - as much as $700-1,000 \mathrm{~m}^{2} / \mathrm{g}$ material.

CPFA is a therapeutic tool that works based on plasma adsorption to provide depurative support for removing excess cytokines, toxins and inflammatory mediators. It also provides support for volume control and small solute removal by the second haemofilter used for CVVH. The cartridge contains a hydrophobic divinylbenzene styrenic resin that was chosen based on removal of many different types of mediators including Interleukin $1 \beta$ (IL-1 $\beta$ ), tu- mor necrosis factor alpha (TNF- $\alpha$ ), IL-6, IL-8, C3a des Arg and IL-10 (Fig. 2). The choice of using plasma filtration instead of haemoperfusion was due to in vitro experiments where it was observed that for some cytokines, there was better binding at lower flow rates [1]. Patient blood passes through the cartridge at a much higher flow rate during haemoperfusion compared to plasma perfusion.

The concept of flow rate is important in any type of adsorptive process using a cartridge. The linear velocity is the term that describes the relationship between the fluid speed and the flow path. It is directly related to the sample flux through the column and the column radius [8].

$$
\text { Linear velocity }=\frac{\text { flux } \mathrm{cm}^{3} / \mathrm{h}}{\text { area } \mathrm{cm}^{2}}
$$

Some resins have good adsorption of a particular molecule over a wide range of linear velocities, while others may have a dramatic decrease in adsorption as the linear velocity increases. This translates into an important point related to plasma adsorption: there is a balance between the volume of plasma being treated and the plasma 
Fig. 3. Adsorption can occur by passing either whole blood or plasma through the sorbent cartridge. Haemoperfusion is associated with a higher blood flow passing through the cartridge (a). This gives the advantage of having a higher volume of blood treated, but there is usually a much more limited adsorption. The fast flow causes several molecules that would normally be adsorbed to be pulled by the solvent flow. In some cases, there are also more problems with surface fouling and cell activation. If the plasma to passes through the cartridge (b), there is longer contact time and this gives greater adsorption efficiency.

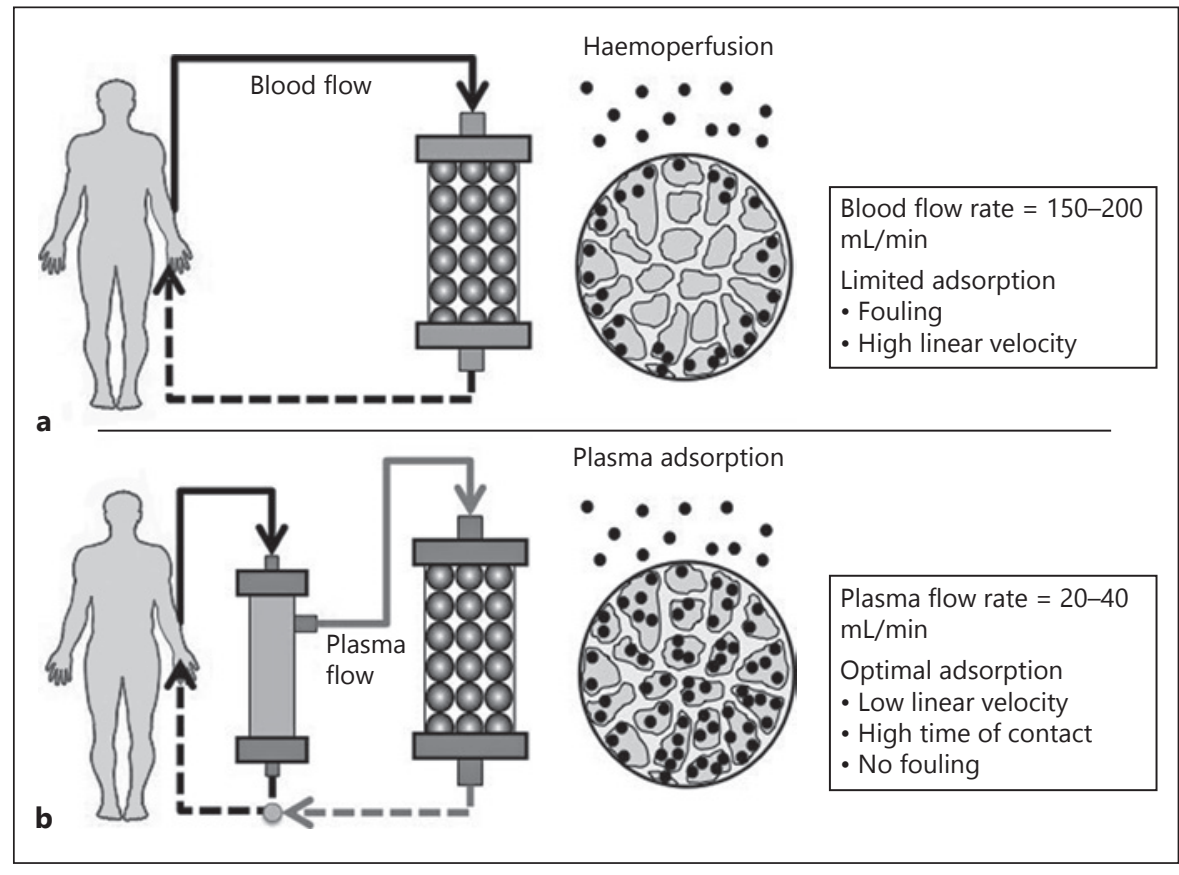

contact time with resin (Fig. 3). Decreased adsorption efficacy is often observed if plasma flow rate becomes too fast [8].

Many cytokines do not have sufficient time for binding to the resin at high flow rates, or in some cases, there is good binding at the start of a session, but this can decrease after a short time due to protein fouling on the resin surface. The CPFA cartridge was optimized for both flow and geometry for adsorption over extended periods of treatment time, and to avoid direct blood contact with the resin, which can sometimes lead to excess protein deposition/fouling and cell activation.

Typically, the session time for CPFA is $10-12 \mathrm{~h}$ with a minimum amount of plasma treated $0.2 \mathrm{~L}$ of plasma/ $\mathrm{kg}$ /day based on ideal body weight. This dose of minimum plasma treated was based on previous studies that appeared to show a benefit for this minimum amount [9-11]. Typical blood flow rates for CPFA are approximately $150-200 \mathrm{~mL} / \mathrm{min}$, whereas the plasma flow is dependent on a fractional filtration of $10-22 \%$ of the blood flow, finally the convective clearance of low and middle molecules takes place through the haemofilter and a filtration fraction less than $25 \%$ is required (Table 1).

The technique should be considered a supportive therapy for depuration of cytokines and needs to be used with appropriate standard of care (antibiotics, appropriate fluid support, other organ support) according to best clinical practice for critically ill patients. Although CPFA is commonly included in the terminology and discussions of continuous renal replacement therapy, it does not need to be limited to patients with AKI. Instead, it is commonly used for clinical syndromes such as sepsis, septic shock, non-infectious severe SIRS, where there is the clinical judgement that removal of excess cytokines may be useful.

\section{CPFA During Septic Shock}

The first in vivo experience of CPFA was done in a rabbit model of endotoxemic shock that evaluated survival with or without CPFA [12]. Rabbits were divided into 4 groups consisting of CPFA or a sham circuit, with or without endotoxin administration. The authors observed a significant increase in 72-h cumulative survival in rabbits administered endotoxin and treated with CPFA compared to rabbits that received endotoxin but were treated with a sham circuit.

This was followed by a pilot study to determine whether there would be a potential benefit for patients with septic shock. Ten patients with septic shock were randomly allocated to either CPFA or CVVHDF. Although both techniques showed an improvement of the mean arterial pressure, patients treated with CPFA had a greater improvement in haemodynamic stability. In addition, the 
Table 1. Steps for the CPFA prescription

\begin{tabular}{|c|c|c|}
\hline Main steps & Prescriptions & Monitoring \\
\hline (A) Anticoagulation & $\begin{array}{l}\text { (1) Regional citrate-calcium } \\
\text { (1.1) Citrate free } \\
\text { (1.2) Citrate assisted } \\
\text { (2) Unfractioned heparin } \\
\text { (2.1) } 10,000 \text { IU in the washing liquid of the circuit } \\
\text { and } 10-15 \mathrm{IU} / \mathrm{Kg} / \mathrm{h} \text { as a maintenance dose } \\
\text { (3) Regional heparin and } 75 \% \text { heparin } \\
\text { neutralization with Protamine } \\
\text { (4) Mixed (citrate + low dose heparin) }\end{array}$ & $\begin{array}{l}\text { 1) Systemic } \mathrm{Ca}++ \\
\text { 2) Total calcium/Ca++<2.5 } \\
\text { 3) Lactates } \\
\text { 4) Electrolytes } \\
\text { (1) Antithrombin III }>80 \% \\
\text { (2) aPTT }<60 \mathrm{~s} \\
\text { (3) ACT }(140-200 \mathrm{~s})\end{array}$ \\
\hline (B) Blood flow rate & $100-200 \mathrm{~mL} / \mathrm{min}$ & $\begin{array}{l}\text { (1) Arterial blood pressure } \\
\text { (2) Dynamic pressures of the blood circuit } \\
\text { (3) Vascular access }\end{array}$ \\
\hline $\begin{array}{l}\text { (C) Plasma filtration } \\
\text { fraction }\end{array}$ & $\begin{array}{l}\text { (1) } 13 \% \text { from CPFA start until the } 2 \text { nd h } \\
\text { (2) } 15 \% \text { from the } 2 \text { nd until the } 4 \text { th } h \\
\text { (3) } 18 \% \text { from the } 4 \text { th until the } 8 \text { th } h \\
\text { (4) From the } 8 \text { th h it is based on the } \\
\text { centre experience or proceed with } \\
\text { CVVH alone }\end{array}$ & $\begin{array}{l}\text { (1) Plasmafilter transmembrane pressure } \\
<20 \mathrm{~mm} \mathrm{Hg} \\
\text { (2) Transcartridge pressure }<150-180 \mathrm{~mm} \mathrm{Hg} \\
\text { (3) Exclude blood leakage detection alarm when use } \\
\text { CPFA for liver failure after dipstick analysis of } \\
\text { the plasma to distinguish between bilirubin and } \\
\text { blood }\end{array}$ \\
\hline $\begin{array}{l}\text { (D) Convective flow } \\
\text { filtration fraction }\end{array}$ & Below 25\% & Haemofilter transmembrane pressure \\
\hline (E) Session time & $\begin{array}{l}10 \mathrm{~h} \\
6 \mathrm{~h}\end{array}$ & $\begin{array}{l}\text { (1) Septic shock, all cases with acute renal failure } \\
\text { (2) Liver failure, all cases without renal failure }\end{array}$ \\
\hline (F) Weight & $\begin{array}{l}\text { (1) Loss } \\
\text { (2) Gain } \\
\text { (2) Euvolemic }\end{array}$ & $\begin{array}{l}\text { (1) Weight scale bed } \\
\text { (2) Convective flow filtration fraction }\end{array}$ \\
\hline
\end{tabular}

authors evaluated immunoresponsiveness of healthy monocytes to incubation with plasma at the entrance to the adsorbent cartridge or immediately after the cartridge at different time points. Incubation of the monocytes with post-cartridge patient plasma, particularly at the end of the 10-h session, was associated with a restoration of the monocytes to produce tumor necrosis factor alpha upon stimulation with lipopolysaccharides [13]. Several later subsequent studies also observed an improvement in haemodynamic parameters with CPFA $[14,15]$.

Based on encouraging small studies, a group of Italian intensivists, GiViTI, decided to do a large randomized multicentre interventional study, COMPACT, to evaluate hospital mortality in septic shock patients by comparing patients treated with CPFA vs. standard of care [10]. This trial was stopped due to technical difficulties associated with the technique. Nearly $50 \%$ of the patients did not reach the target amount of plasma (primarily due to coagulation of the circuit). Although the trial did not see any statistical difference in hospital mortality be- tween the 2 groups, they did observe what appeared to be a plasma-dose related benefit for survival in the patients that received the full amount of plasma treatment (at least $0.2 \mathrm{~L}$ plasma $/ \mathrm{kg} /$ day) from an a priori planned subgroup analysis.

This prompted 2 additional randomized controlled studies: ROMPA - a multicentre interventional CPFA study based in Spain, which is still ongoing [16], and a second study, COMPACT2, by the same group of Italian physicians, which has an adaptive study design to evaluate technical feasibility to achieve high doses of treated plasma, days free of septic shock and mortality.

One of the major concerns about using any blood purification therapy to treat critically ill patients is that there is concern that not enough is known about the underlying pathophysiology and the target for removal. A recent study by Castellano et al. [17] tried to address this issue with the use of a swine model to study the pathophysiology of endotoxin-induced AKI. They infused LPS and observed acute tubulointerstitial fibrosis and endothelial 
Fig. 4. Rational of CPFA application during

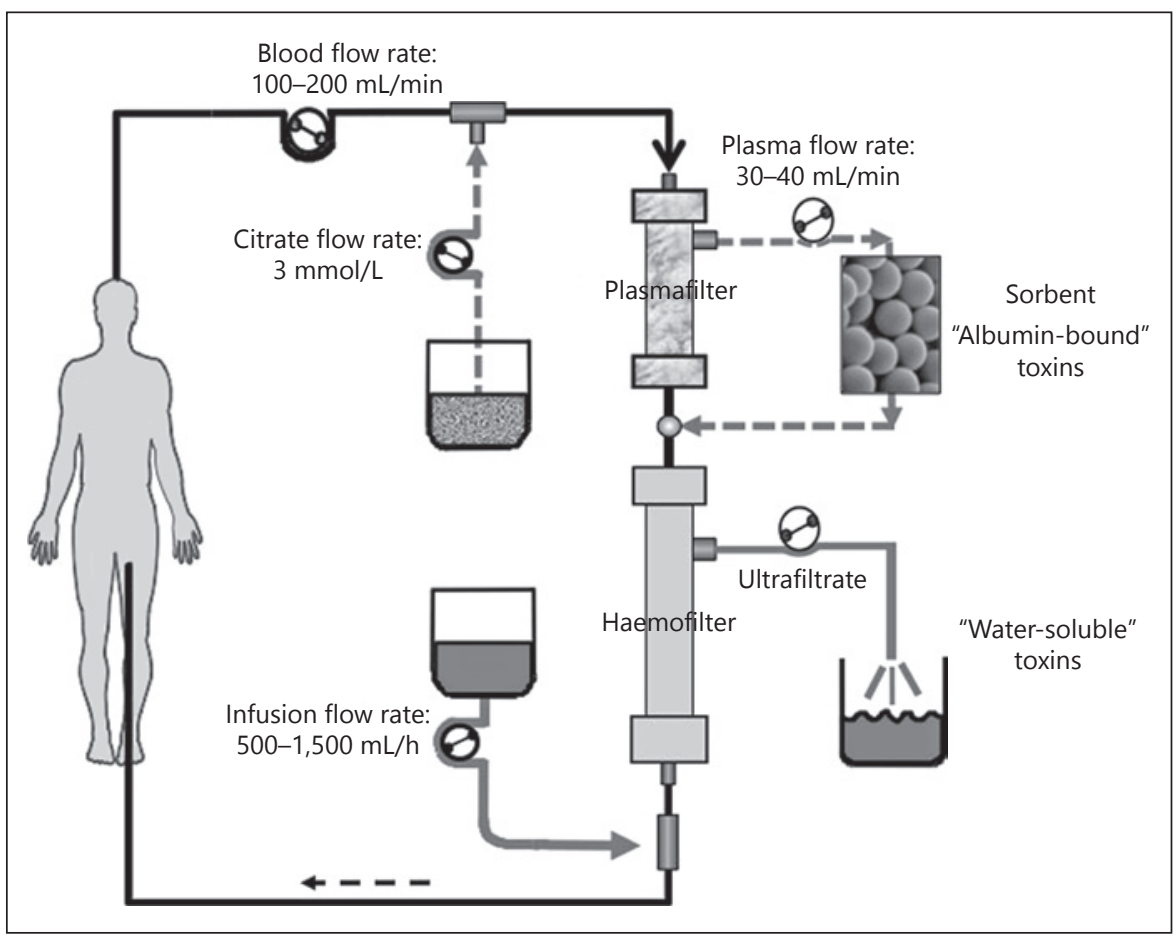
liver dysfunction.

Table 2. Toxins retained during liver failure, modified from Laleman et al. [34]

\begin{tabular}{ll}
\hline Water-soluble & Albumin-bound \\
\hline Low molecular weight & Bilirubin \\
Ammonia & Bile acids \\
Lactate & Aromatic amino acids \\
Urea & Digoxin-like substances \\
Medium molecular weight & Endogenous benzodiazepines \\
Amino acids & Indols \\
Peptides & Mercaptans \\
GABA & Nitric oxide \\
Interleukins & Middle- and short- chain fatty acids \\
& Phenols \\
& Prostacyclins \\
& Tryptophan \\
& Manganese \\
& TNF- $\alpha$ \\
\end{tabular}

dysfunction that occurred within $9 \mathrm{~h}$ of LPS injection. Treatment with $6 \mathrm{~h}$ of CPFA significantly prevented acute fibrosis by preserving the endothelial phenotype in both peritubular capillaries and renal arteries. Their work suggested that the mechanism for this was due to the removal of the lipopolysaccharide-binding protein from the plasma, which was shown to have significant adsorption by the CPFA cartridge [17].

\section{CPFA and Liver Dysfunction}

CPFA has also been used to remove toxins associated with liver dysfunction (Table 2, Fig. 4). Although traditional haemodialysis or haemofiltration are effective techniques for clearance of ammonia and small molecules, they are not effective for the removal of proteinbound toxins [18]. Harm et al. [19] reported that the hydrophobic polystyrene-divinylbenzene copolymer of CPFA resin cartridge (type B copolymer: mean diameter 128 micron, pore size 30 nanometres and surface area of $700 \mathrm{~m}^{2} / \mathrm{g}$ of resin) binds in vitro higher amount of bilirubin than the amount of bilirubin bound with the other kinds of polystyrene-divinylbenzene copolymer matrices tested (type A copolymer: mean diameter 120 micron, pore size 15 nanometres and surface area of $900 \mathrm{~m}^{2} / \mathrm{g}$ of resin; type $\mathrm{C}$ copolymer: mean diameter 37 micron, pore size 100 nanometres and surface area of $200 \mathrm{~m}^{2} / \mathrm{g}$ of resin). Nonetheless, the same resin achieves an optimal in vitro detoxification for tryptophan and phenols, 2 important protein-bound toxins that act as false neurotransmitters related to the hepatic encephalopathy [19]. As a consequence, CPFA might be employed (a) as a bridge to the recovery of basal liver function or to liver transplantation in case of acute or acute on chronic liver failure; (b) for the prevention of bile cast nephropathy in case of total bilirubin values $\geq 20 / \mathrm{dL}$ [20]. 
Table 3. CPFA during liver dysfunction

\begin{tabular}{lcclc}
\hline Authors & Patients, $n$ & CPFA sessions, $n$ & Cause of liver dysfunction & $\begin{array}{c}\text { Bilirubin reduction } \\
\text { ratio (mean \%) }\end{array}$ \\
\hline Caroleo et al. [22] & 1 & 3 & Acute liver failure & 53 \\
Donati et al. [23] & 1 & 2 & Acute liver failure & 40 \\
Maggi et al. [21] & 2 & 6 & OLT primary non function & 40 \\
Ullo et al. [24] & 9 & 22 & Acute or acute on chronic liver failure & 28 \\
De Simone et al. [25] & 10 & 34 & Obstructive cholangiocarcinoma & 30 \\
\hline
\end{tabular}

A few authors also assessed the in vivo detoxification ability of CPFA during liver dysfunction (Table 3). Maggi et al. [21] reported their experience on using CPFA for 2 post-liver transplant patients that developed allograft dysfunction and hyperbilirubinemia linked to chronic rejection [21]. They used 3 treatment cycles lasting $6 \mathrm{~h}$. In the first case, the high bilirubin levels were the expression of early allograft dysfunction in a patient that had received a marginal liver due to steatosis. In the second patient, the patient displayed late hyperbilirubinemia with signs of mild acute rejection that evolved to chronic rejection. In both cases there was a marked bilirubin reduction (approximately 40\%) over the course of treatment and patients were successfully discharged from the hospital. The second patient, however, had a much poorer overall prognosis and eventually died later of other complications. The authors suggested that prognosis should be one of the key points to decide whether or not to use CPFA, if this is a bridge to other definitive therapies.

Caroleo et al. [22] also reported their experience of treating a 70-year-old woman who developed hypoxic hepatitis secondary to cardiogenic shock. The patient was admitted to the ICU after complications from cardiac surgery. She further developed AKI and a dramatic increase in serum aminotransaminase activity and hyperbilirubinemia from hypoxic hepatitis [22]. CPFA was used to remove inflammatory mediators and reduce bilirubin and fluid overload. In addition to beneficial renal and haemodynamic effects, they observed a removal rate for conjugated bilirubin of $47.8 \%$ after the first CPFA cycle and $53.8 \%$ after the second cycle and 59.3\% after the third cycle.

Our group [23] carried out successfully 2 CPFA treatments, 6-h length, in a patient with acute liver failure with jaundice and encephalopathy. The bilirubin reduction rate per each session was $41 \%$. In the latter case, the patient started CPFA with a total bilirubin value of
$71 \mathrm{mg} / \mathrm{dL}$, and $8 \mathrm{~L}$ of plasma was detoxificated for each CPFA session. Currently, there is an independent observational study (HERCOLE, ClinicalTrials.gov ID: NCT03312036) that will try and address the question as to whether there is a survival benefit and a detoxification efficacy of CPFA for using 6-h length CPFA in patients with acute or acute on chronic hepatic failure [24]. An interim analysis after the enrolment of 9 patients assessed that at 1 year of follow-up, 2 patients died during the hospitalization; 7 patients were followed like outpatients and 1 of them is no more in the waiting list for liver transplantation. A total of 22 CPFA treatments were carried out. As to the detoxification efficacy, the median reduction rate per CPFA session for total bilirubin was $28 \%$ (range 2.2-40); for direct bilirubin was 31.4 (range 8.5-48.6); for indirect bilirubin was $29.1 \%$ (range 6.6$65)$; and for bile acids was 30.6\% (16.7-59.6). De Simone et al. [25] confirmed that CPFA therapy is an effective detoxification therapy for hyperbilirubinemia in 10 patients affected by hilar cholangiocarcinoma complicated with obstructive jaundice. Thirty-four CPFA treatments were carried out and CPFA reduced preoperative bilirubin by $30 \%$ [25].

\section{Emerging Additional Indications for CPFA}

Although the most common experience for CPFA has been in the applications of sepsis and septic shock, the resin in the plasma cartridge is also able to remove cytokines in various systemic inflammatory states, myoglobin, various toxins and some drugs associated with overdose.

Conventional haemodialysis is not able to remove myoglobin efficiently and high circulating levels can lead to further glomerular and renal tubule damage [26]. The resin in the CPFA cartridge has a high affinity for myoglobin associated with rhabdomyolysis. Lai et al. 
[27] recently published a report of 2 case series where they used CPFA in 2 kidney transplant patients with severe unexplained rhabdomyolysis. In both cases, they had a marked reduction in myoglobin and an improvement in many inflammatory parameters such as CRP. They suggested the CPFA may have had a role in the improvement of the clinical situation of the patients by (1) the adsorption of myoglobin, (2) removal of inflammatory mediators and (3) management of AKI in this setting with the addition of the simultaneous use of CVVH.

Another case series report by Pezzi et al. [28] was recently published reporting the use of CPFA for traumatic rhabdomyolysis in 4 patients with AKI. They used CPFA for $10 \mathrm{~h}$ followed by CVVH for $14 \mathrm{~h}$. Two patients underwent 3 treatments in $72 \mathrm{~h}$, while the other 2 patients underwent 4 treatments in $96 \mathrm{~h}$. They observed a marked decrease in myoglobin and creatinine kinase and an improvement in renal function for all 4 patients after each CPFA session and at the end of the 3rd or 4th treatment.

Another example of a very complex case treated with CPFA was that of a morbidly obese 77-year-old woman with post-vaccination complications of Guillain-Barre' [29]. The patient was treated with IV immunoglobulin, but she experienced complications of hyperthermia, cardiac arrest and septic shock. The patient underwent $2 \mathrm{~h}$ of cardiopulmonary resuscitation and was haemodynamically unstable. CPFA was started for $10 \mathrm{~h}$ followed by $12 \mathrm{~h}$ of CVVH. At the end of the treatment, there was a significant improvement in inflammatory markers and d-dimer. After 36 days, the patient was discharged with full ability to spontaneously move all limbs and was without any sensory or motor deficit. The authors suggested that the good clinical response obtained with the application of the CPFA in this patient has contributed to positive effects both on the septic shock and on the inflammatory demyelinising polyneuropathy.

A case of CPFA application during thyreotoxicosis to achieve free triiodothyronine (FT3) and free thyroxin (FT4) adsorption was also reported by our group [23]. Two CPFA sessions were carried out with $8 \mathrm{~L}$ of plasma detoxificated for each CPFA session. The resin saturation with FT3 or FT4 was checked by means of measuring the plasma FT3 and FT4 levels before and after the cartridge every hour from CPFA start for 7 times until the CPFA end. The resin of CPFA allowed the adsorption of FT3, with a reduction rate per session between 30 and $36 \%$. The FT3 post cartridge values re- mained lower than the pre-cartridge FT3 values also after $6 \mathrm{~h}$ indicating that there was no saturation of the resin after $6 \mathrm{~h}$ of treatment. This would potentially allow even longer treatments for FT3 removal. Nonetheless, the resin also showed a high affinity with rapid saturation for FT4 because after $4 \mathrm{~h}$ of treatment, the post cartridge FT4 values approached the pre-cartridge values. Such behaviour did not cause a significant reduction in the circulating FT4 values, indicating a rapid shift from the bound T4 pool to the free T4 fraction. Nonetheless, in the same case report, CPFA allowed a significant reduction rare per session of amiodarone plasma levels of $35 \%$.

\section{Future of CPFA and Evolution}

Anticoagulation remains an important aspect for success of blood purification techniques. CPFA has recently undergone several modifications to improve the technical aspects and citrate anticoagulation with an increased focus on protocols, software and machine improvements to facilitate the use of both heparin and citrate. Mariano et al. [30] assessed a rather higher than typical circuit citratemia of $6 \mathrm{mmol} / \mathrm{L}$ in both HDF predilution and HF pre/postdilution and found that the best dialysis modalities to maintain a normal systemic citratemia are through a high rate of citrate loss in the effluent in septic shock patients.

Other groups have approached hybrid or modified systems to try and further eliminate both cytokines and bacterial components such as endotoxin. An example of this is the work of Yaroustovsky et al. [31] that combined CPFA with LPS adsorption by the Toraymyxin columns and carried out 40 extracorporeal treatments in 20 patients with severe sepsis after cardiac surgery. The 28 -day survival rate was $65 \%$ in the study group and $35 \%$ in the control group. In contrast to controls, patients who underwent CPFA + Toraymyxin experienced a significant increase of mean arterial pressure and of the mean oxygenation index and a significant decrease of endotoxemia and of procalcitonin levels.

A future approach might be the CPFA use also in case of exogenous poisoning: in case of poisons with high protein binding (e.g., amiodarone [23]) the resin competes with albumin for binding and removes the protein-poison complexes [32]. Previously, Berning et al. [33] also observed the effectiveness of plasma perfusion with resins in the elimination of toxic substances: after the separation 
from blood, plasma was perfused on the resin adsorber. Thirty patients underwent plasma perfusion and during each treatment, a mean volume of $3,800 \mathrm{~mL}$ of plasma was treated and returned to the patients. No side effects were reported [33].

In conclusion, the main advantage of CPFA is its ability to treat a higher amount of plasma without plasma or albumin replacement and with a high haemodynamic tolerance. The role of the haemofilter is intended to enhance the haemodynamic tolerance of the extracorporeal treatment and remove water-soluble toxins in the range of the medium molecules. Combining plasma adsorption and haemofiltration is useful, especially for those toxins with a high volume of distribution that can benefit from a prolonged extracorporeal detoxification. Nonetheless, the depurative efficiency of CPFA might be further improved, in particular with regard to increased surface area of the resin, and the possibility to tailor more specific resins to target different toxins and mediators.

\section{Disclosure Statement}

The authors declare that they have no conflicts of interest to disclose.

\section{References}

1 Tetta C, Cavaillon JM, Schulze M, Ronco C, Ghezzi PM, Camussi G, Serra AM, Curti F, Lonnemann G: Removal of cytokines and activated complement components in an experimental model of continuous plasma filtration coupled with sorbent adsorption. Nephrol Dial Transplant 1998;13:1458-1464.

2 Ronco C, Tetta C, Mariano F, Wratten ML Bonello $\mathrm{M}$, Bordoni $\mathrm{V}$, Cardona $\mathrm{X}$, Inguaggiato P, Pilotto L, d'Intini V, Bellomo R: Interpreting the mechanisms of continuous renal replacement therapy in sepsis: the peak concentration hypothesis. Artif Organs 2003;27: 792-801.

3 Bone RC: Immunologic dissonance: a continuing evolution in our understanding of the systemic inflammatory response syndrome (SIRS) and the multiple organ dysfunction syndrome (MODS). Ann Intern Med 1996; 125:680-687.

4 Angus DC, van der Poll T: Severe sepsis and septic shock. N Engl J Med 2013;369:840-851.

5 Van der Poll T: Immunotherapy of sepsis. Lancet Infect Dis 2001;1:165-174.

6 Ward PA: New approaches to the study of sepsis. EMBO Mol Med 2012;4:1234-1243.

7 Oyama Y, Takeda T, Hama H, Tanuma A, Iino N, Sato K, Kaseda R, Ma M, Yamamoto T, Fujii H, Kazama JJ, Odani S, Terada Y, Mizuta K, Gejyo F, Saito A: Evidence for megalin-mediated proximal tubular uptake of L-FABP, a carrier of potentially nephrotoxic molecules. Lab Invest 2005;85:522531.

8 Ronco C, Brendolan A, d'Intini V, Ricci Z, Wratten ML, Bellomo R: Coupled plasma filtration and adsorption: rationale, technical development and early clinical experience. Blood Purif 2003;21:409-416.

9 Berlot G, Agbedjro A, Tomasini A, Bianco F, Gerini U, Viviani M, Giudici F: Effects of the volume of processed plasma on the outcome, arterial pressure and blood procalcitonin levels in patients with severe sepsis and septic shock treated with coupled plasma filtration and adsorption. Blood Purif 2014;26;37:146151.

10 Livigni S, Bertolini G, Rossi C, Ferrari F, Giardino M, Pozzato M, Remuzzi G: Efficacy of coupled plasma filtration adsorption (CPFA) in patients with septic shock: a multicenter randomised controlled clinical trial. BMJ Open 2014;4:e003536.

11 Kellum JA: Renal replacement therapy in critically ill patients with acute renal failure: does a greater dose improve survival? Nat Clin Pract Nephrol 2007;3:128-129.

12 Tetta C, Gianotti L, Cavaillon JM, WrattenML, Fini M, Braga M, Bisagni P, Giavaresi G, Bolzani R, Giardino R: Coupled plasma filtration-adsorption in a rabbit model of endotoxic shock. Crit Care Med 2000;28:1526-1533.

13 Ronco C, Brendolan A, Lonnemann G, Bellomo R, Piccinni P, Digito A, Dan M, Irone M, La Greca G, Inguaggiato $P$, Maggiore U, De Nitti C, Wratten ML, Ricci Z, Tetta C: A pilot study of coupled plasma filtration with adsorption in septic shock. Crit Care Med 2002; 30:1250-1255.

14 Franchi M, Giacalone M, Traupe I, Rago R, Baldi G, Giunta F, Forfori F: Coupled plasma filtration adsorption improves hemodynamics in septic shock. J Crit Care 2016;33:100105.

15 Formica M, Olivieri C, Livigni S, Cesano G, Vallero A, Maio M, Tetta C: Hemodinamic response to coupled plasmafiltration-adsorption in human septic shock. Intensive Care Med 2003;29:703-708.

16 Colomina-Climent F, Giménez-Esparza C, Portillo-Requena C, Allegue-Gallego JM, Galindo-Martínez M, Mollà-Jiménez C, Antón-Pascual JL, Rodríguez-Serra M, MartínRuíz JL, Fernández-Arroyo PJ, Blasco-Císcar EM, Cánovas-Robles J, Herrera-Murillo M, González-Hernández E, Sánchez-Morán F, Solera-Suárez M, Torres-Tortajada J, Nuñez-Martínez JM, Martín-Langerwerf D,
Herrero-Gutiérrez E, Sebastián-Muñoz I, Palazón-Bru A, Gil-Guillén VF: Mortality reduction in septic shock by plasma adsorption (ROMPA): a protocol for a randomised clinical trial. BMJ Open 2016; 6:e011856.

17 Castellano G, Stasi A, Intini A, Gigante M, Di Palma AM, Divella C, Netti GS, Prattichizzo C, Pontrelli P, Crovace A, Staffieri F, Fiaccadori E, Brienza N, Grandaliano G, Pertosa G, Gesualdo L: Endothelialdysfunction and renalfibrosis in endotoxemia-inducedoligurickidneyinjury: possiblerole of LPS-bindingprotein. Crit Care 2014;18:520.

18 Slack AJ, Auzinger G, Willars C, Dew T, Musto R, Corsilli D, Sherwood R, Wendon JA, Bernal W: Ammonia clearance with haemofiltration in adults with liver disease. Liver Int 2014;34:42-48.

19 Harm S, Falkenhagen D, Hartmann J: Pore size - a key property for selective toxin removal in blood purification. Int J Artif Organs 2014;37:668-678.

20 Van Slambrouck CM, Salem F, Meehan SM, Chang A: Bile cast nephropathy is a common pathologic finding for kidney injury associated with severe liver dysfunction. Kidney Int 2013;84:192-197.

21 Maggi U, Nita G, Gatti S, Antonelli B, Paolo R, Como G, Messa P, Rossi G: Hyperbilirubinemia after liver transplantation: the role of coupled plasma filtration adsorption. Transplant Proc 2013;45:2715-2717.

22 Caroleo S, Rubino AS, Tropea F, Bruno O, Vuoto D, Amantea B, Renzulli A: Coupled plasma filtration adsorption reduces serum bilirubine in a case of acute hypoxic hepatitis secondary to cardiogenic shock. Int J Artif Organs 2010;33:749-752.

23 Donati G, Capelli I, Croci Chiocchini AL, Natali N, Scrivo A, La Manna G: Coupled plasma filtration adsorption application for liver and thyroid toxins. Contrib Nephrol 2017;190: $31-42$. 
24 Ullo I, Zappulo F, Bini C, Bruno P, Scrivo A, Donati G, Bolondi L, Piscaglia F, Simoni P, La Manna G: Coupled plasma filtration and adsorption (CPFA) for extracorporeal detoxification during acute and acute onchronic liver failure. Blood Purif 2017;44: 182.

25 De Simone W, Crafa F, Noviello A, Esposito F, Zito B, Manganelli R, De Simone A, Covotta L, Palladino G, De Simone E: [Bilirubin removal with coupled plasma filtration and adsorption in patients affected by hilar cholangiocarcinoma]. G Ital Nephrol 2017;34:pii: 2017-vol6.

26 Vanholder R, Sever MS, Erek E, Lameire N: Rhabdomyolysis. J Am Soc Nephrol 2000;11: 1553-1561.

27 Lai Q, Di Pietro V, Iesari S, Amabili S, De Luca L, Clemente K, Famulari A, Pisani F: Coupled plasma filtration adsorption in patients with a history of kidney transplanta- tion: report of two cases. Blood Purif 2015;40: 218-222.

28 Pezzi M, Renda S, Giglio AM, Scozzafava AM, Tiburzi SP, Casella P, Iannelli F, Verre M: The use of coupled plasma filtration adsorption in traumatic rhabdomyolysis. Case Rep Crit Care 2017;2017:5764961.

29 Fabbri LP, Macchi A, Lecchini R, Pennacchi A: Post influenza vaccination Guillain-Barrésyndrome: an exceptional recovery case aftercardiac arrest for two hours in hyperthermia and septic shock. Anaest Pain Intensive Care 2015;19:166-169.

30 Mariano F, Morselli M, Holló Z, Agostini F, Stella M, Biancone L: Citrate pharmacokinetics at high levels of circuit citratemia during coupled plasma filtration adsorption. Nephrol Dial Transplant 2015;30:1911-1919.

31 Yaroustovsky M, Abramyan M, Krotenko N, Popov D, Plyushch M, Rogalskaya E: A pilot study of selective lipopolysaccharide adsorp- tion and coupled plasma filtration adsorption in adult patients with severe sepsis. Blood Purif 2015;39:210-217.

32 Ghannoum M, Roberts DM, Hoffman RS, Ouellet G, Roy L, Decker BS, Bouchard J: A stepwise approach for the management of poisoning with extracorporeal treatments. Semin Dial 2014;27:362-370.

33 Berning T, Krummenerl T, Glaser J, Paulus H, Gerlach U: [Plasma perfusion in life-threatening exogenous poisoning]. Schweiz Med Wochenschr 1987;117:1368-1373.

34 Laleman W, Wilmer A, Evenepoel P, Verslype C, Fevery J, Nevens F: Review article: non-biologic liver support in liver failure. Aliment Pharmacol Ther 2006;23:351363.

35 Bello G, Di Muzio F, Maviglia R, Antonelli M: New membranes for extracorporeal blood purification in septic conditions. Miner Anestesiol 2012;78:1265-1281. 